\title{
Electroporation-mediated RNA Interference Method in Odonata
}

\author{
Genta Okude ${ }^{1,2}$, Takema Fukatsu ${ }^{1,2,3}$, Ryo Futahashi ${ }^{2}$ \\ ${ }^{1}$ Department of Biological Sciences, Graduate School of Science, The University of Tokyo ${ }^{2}$ Bioproduction Research Institute, National Institute of \\ Advanced Industrial Science and Technology (AIST) ${ }^{3}$ Graduate School of Life and Environmental Sciences, University of Tsukuba
}

\section{Corresponding Authors}

Genta Okude

gentaokude@gmail.com

Ryo Futahashi

ryo-futahashi@aist.go.jp

\section{Citation}

Okude, G., Fukatsu, T.

Futahashi, R. Electroporation-

mediated RNA Interference Method in

Odonata. J. Vis. Exp. (168), e61952,

doi:10.3791/61952 (2021).

\section{Date Published}

February 6, 2021

\section{DOI}

$10.3791 / 61952$

\section{URL}

jove.com/video/61952

\section{Abstract}

Dragonflies and damselflies (order Odonata) represent one of the most ancestral insects with metamorphosis, in which they change their habitat, morphology, and behavior drastically from aquatic larvae to terrestrial/aerial adults without pupal stage. Odonata adults have a well-developed color vision and show a remarkable diversity in body colors and patterns across sexes, stages, and species. While many ecological and behavioral studies on Odonata have been conducted, molecular genetic studies have been scarce mainly due to the difficulty in applying gene functional analysis to Odonata. For instance, RNA interference (RNAi) is less effective in the Odonata, as reported in the Lepidoptera. To overcome this problem, we successfully established an RNAi method combined with in vivo electroporation. Here we provide a detailed protocol including a video of the electroporation-mediated RNAi method as follows: preparation of larvae, species identification, preparation of dsRNA/siRNA solution and injection needles, ice-cold anesthesia of larvae, dsRNA/siRNA injection, in vivo electroporation, and individual rearing until adult emergence. The electroporationmediated RNAi method is applicable to both damselflies (suborder Zygoptera) and dragonflies (suborder Anisoptera). In this protocol, we present the methods for the blue-tailed damselfly Ischnura senegalensis (Coenagrionidae) as an example of damselfly species and the pied skimmer dragonfly Pseudothemis zonata (Libellulidae) as another example of dragonfly species. As representative examples, we show the results of RNAi targeting the melanin synthesis gene multicopper oxidase 2 . This RNAi method will facilitate understanding of various gene functions involved in metamorphosis, morphogenesis, color pattern formation, and other biological features of Odonata. Moreover, this protocol may be generally applicable to non-model organisms in which RNAi is less effective in gene suppression due to the inefficiency and low penetrance. 


\section{Introduction}

Dragonflies and damselflies (the order Odonata) are among the most ancestral groups of insects that exhibit "metamorphosis"1,2. By metamorphosis, they change their habitat, morphology, and behavior drastically from aquatic larvae to terrestrial/aerial adults ${ }^{3}$. Odonata adults have a welldeveloped color vision and represent a remarkable diversity in body colors and patterns across sexes, stages, and species $^{3,4,5}$. While many ecological and behavioral studies on Odonata have been conducted $^{6,7}$, molecular genetic studies have been hindered mainly by the difficulty in applying gene functional analysis to Odonata.

The conventional RNA interference (RNAi) method, in which double-stranded RNA (dsRNA) is injected to suppress the function of the gene of interest ${ }^{8}$, turned out to be ineffective in Odonata insects ${ }^{9}$, as reported in Lepidopteran insects ${ }^{10}$. On the other hand, previous reports have suggested that electroporation-mediated RNAi is effective in Lepidopteran species, especially in epidermal tissues ${ }^{11,12,13}$. We recently found that the electroporation-mediated RNAi works effectively in the tiny dragonfly Nannophya pygmaea (Libellulidae: Anisoptera) ${ }^{9}$, but $N$. pygmaea is a relatively rare species and therefore not suitable for molecular genetic studies.

Most Odonata species are classified into either of the two suborders, Zygoptera (damselflies) or Anisoptera (true dragonflies $)^{3}$. Here we focused on the blue-tailed damselfly Ischnura senegalensis (Coenagrionidae; Figure 1A) as a representative Zygopteran species and the pied skimmer dragonfly Pseudothemis zonata (Libellulidae; Figure 1B) as a representative Anisopteran species. The two species are among the most common Odonata species in natural and urban ponds in Japan, including those in Tsukuba City, and we can collect many larvae of the two species in the field. Recently we established a laboratory rearing system for individual larvae of $I$. senegalensis, which enabled continuous monitoring of development and morphogenesis of the Odonata larvae in detail ${ }^{14}$.

In this report, we provide a refined method and a video protocol for the electroporation-mediated RNAi in $I$. senegalensis and P. zonata. In Japan, I. senegalensis and Ischnura asiatica, which are genetically close, are often found sympatrically ${ }^{15}$, and they are difficult to distinguish in larvae ${ }^{16}$. We also describe how two Ischnura species can be distinguished by restriction fragment length polymorphism (RFLP).

For evaluating the effectiveness of the electroporationmediated RNAi, we select multicopper oxidase 2 gene (MCO2; also known as laccase2) as a representative target gene, on account of the visible phenotype of paler cuticle color upon knockdown of the gene expression ${ }^{9}$. MCO2 is known to be essential for darkening of the epidermis in a variety of insect species ${ }^{17,18}$.

\section{Protocol}

NOTE: The overall scheme of the protocols is shown in Figure 1.

\section{Preparation of larvae of dragonflies or damselflies.}

1. Collect larvae in the field using a hand net.

NOTE: I. senegalensis larvae often cling onto water plants floating on the water surface, while $P$. zonata 
larvae often stay among leaf litter at the bottom. In Tsukuba City, the final instar larvae of $I$. senegalensis are found mainly from March to June, and those of $P$. zonata from May to June. I. senegalensis larvae can be reared from eggs in the laboratory ${ }^{14}$, but the larvae collected in the field have the clearly higher success rate of adult emergence.

2. Species identification by restriction fragment length polymorphism (RFLP) of PCR-amplified products.

NOTE: This step is only necessary if the species is difficult to identify from the appearance of the larvae, as in I. senegalensis.

1. Hold one of the caudal gills of a larva using forceps. Then, the larva falls off its caudal gill itself (causing autotomy).

NOTE: Larvae of Zygopteran damselflies usually have three caudal gills (Figure 1A). When they are attacked by a predator, they can take off their own caudal gills to escape. If the caudal gill is not available, a portion of the leg is dissected.

2. Put one caudal gill into $100 \mu \mathrm{L}$ of $\mathrm{PBS}$ solution $[0.8 \%$ $\mathrm{NaCl}, 0.02 \% \mathrm{KCl}, 0.115 \% \mathrm{Na}_{2} \mathrm{HPO}_{4}$, and $0.02 \%$ $\left.\mathrm{KH}_{2} \mathrm{PO}_{4}(\mathrm{w} / \mathrm{v})\right]$ and homogenize with a hand mixer using a deposit pestle.

3. Spin down the mixture at $5,000 \times g$ for 10 seconds, and subject $0.5 \mu \mathrm{L}$ of the supernatant to PCRamplification using DNA polymerase and primers to amplify the internal transcribed spacer 1 (ITS1) region of the nuclear DNA.

NOTE: Perform the PCR according to the manufacturer's protocol. The combination of ITSF0 (5'- GGA AAG ATG GCC AAA CTT GA -3') and 5.8S-AS1 (5'- GCC GGC CCT CAG CCA G
-3') primers can amplify ITS1 region in almost all Odonata species ${ }^{19}$.

4. Incubate the mixture of $1 \mu \mathrm{L}$ of PCR-amplified product, $0.3 \mu \mathrm{L}$ of $10 \mathrm{x} \mathrm{M}$ Buffer, $0.1 \mu \mathrm{L}$ of Dral restriction enzyme, and $1.6 \mu \mathrm{L}$ of water at $37^{\circ} \mathrm{C}$ for $1 \mathrm{~h}$.

NOTE: Select the best restriction enzymes, depending on the combination of species. If there are no restriction enzymes suitable for species identification, confirm by DNA sequencing.

5. Load $2 \mu \mathrm{L}$ of the products with loading dye on $2 \%$ agarose gel and run electrophoresis.

NOTE: It is difficult to identify species when using $1 \%$ or $1.5 \%$ agarose gel.

6. Check the electrophoresis pattern to identify the species (Figure 2).

NOTE: RFLP patterns are species- and populationdependent. In Tsukuba population, I. asiatica has a major band of 400-500 bp, whereas I. senegalensis has a major band of approximately $200 \mathrm{bp}$ and an additional band of less than $100 \mathrm{bp}$ (an arrowhead in Figure 2). The ITS1 region exists in multiple copies in the genome, and in Ischnura species, microsatellite polymorphism within the ITS1 region is often present in the same individual, affecting the pattern of RFLPs.

3. Rear the collected larvae in the laboratory until use for RNAi.

1. Place each larva separately into each well of a 12well plate with approximately $3 \mathrm{~mL}$ of water.

NOTE: I. senegalensis larvae must be kept individually because they frequently cannibalize 
each other, while $P$. zonata larvae can be kept in a group because they rarely cannibalize.

2. Feed I. senegalensis larvae with Artemia brine shrimp every day and $P$. zonata larvae with bloodworms and/or Tubifex worms at least twice a week until they grow to the suitable developmental stage for RNAi.

NOTE: Frequent feeding is critical to increase the success rate of adult emergence.

3. Change the water as soon as it becomes dirty with feces or leftovers.

NOTE: Frequent water change is also important to increase the success rate of adult emergence.

4. Judge the appropriate developmental stage for RNAi.

NOTE: The final instar larvae of $I$. senegalensis can be classified into five developmental stages ${ }^{14,20}$. The first stage (stage $A$, before the wings begin to expand) or the second stage (stage B) of the final instar larvae is suitable for RNAi experiments, when considering the clear phenotype of RNAi after adult emergence (see Discussion). In $P$. zonata, the final instar larvae before wing expansion (corresponding to stage A of $I$. senegalensis) were used in this study.

\section{Preparation of dsRNA/siRNA solution and injection needles for RNAi.}

NOTE: Select either small interfering RNA (siRNA) (step 2.1) or double-stranded RNA (dsRNA) (step 2.2) as the solution for RNAi.

1. Preparation of the siRNA solution
1. Design siRNA using siDirect program version 2.0 (http://sidirect2.rnai.jp/) ${ }^{21}$,following the guidelines previously reported in Lepidopteran insects ${ }^{22}$.

2. Obtain commercially synthesized siRNA.

NOTE: The sequences of siRNA targeting MCO2 gene of $I$. senegalensis used in this study were as follows: 5'- GCA CUU UCC GUU AUC AAU AUA -3' for sense strand and 5'- UAU UGA UAA CGG AAA GUG CUC -3' for antisense strand. As a negative control, the sequences of siRNA targeting enhanced green fluorescent protein (EGFP) gene were as follows: 5'- GCA UCA AGG UGA ACU UCA AGA -3' for sense strand and 5'- UUG AAG UUC ACC UUG AUG CCG $-3^{\prime}$ for antisense strand ${ }^{11}$.

3. Dilute the siRNA to $100 \mu \mathrm{M}$ with injection buffer [100 mM CH $33 \mathrm{COOK}, 2 \mathrm{mM} \mathrm{Mg}\left(\mathrm{CH}_{3} \mathrm{COO}\right)_{2}, 30 \mathrm{mM}$ HEPES-KOH, $\mathrm{pH} 7.4]^{22}$.

4. Store at $-80^{\circ} \mathrm{C}$ until use.

2. Preparation of dsRNA solution.

1. Select a 300-400 bp region for dsRNA using primer3 program version 4.1 .0 (http://bioinfo.ut.ee/ primer $3 /)^{23}$ and design the primer sets.

2. Obtain commercially synthesized primer sets.

NOTE: To produce templates for dsRNA synthesis, the primer sets used in this study were as follows: (5'- GCC TGT CAG CTT TGT CTT CC -3' for forward primer and 5'- GGT GTC TGG CGG ACA ACT AT -3 ' for reverse primer) for MCO2 genes of $l$. senegalensis (IsMCO2, accession no. LC589180) and (5'- CCG CAC AGC TCA CTA TTC AA -3' for forward primer and 5'- GGA GGA TTC CTT CAT CGA CA $-3^{\prime}$ for reverse primer) for $M C O 2$ genes of 
P. zonata (PzMCO2, accession no. LC589179). As a negative control, the primer set for dsRNA targeting $\beta$-lactamase (bla) gene on the cloning vector were (5'- CTA TGT GGC GCG GTA TTA T -3' for forward primer and 5'- CAG AAG TGG TCC TGC AAC T -3' for reverse primer).

3. Extract RNA from Odonata larvae using a commercially available RNA extraction kit and perform cDNA synthesis using reverse transcriptase according to the manufacturer's protocol.

NOTE: cDNA library prepared for RNA sequencing analysis can be also used.

4. Amplify the target sequences using the synthesized cDNA and the designed primer set and clone them into the cloning vector using a commercial ligase according to the manufacturer's protocol.

5. Transform the plasmid into E. coli competent cells and pick up a single colony after overnight incubation.

6. PCR-amplify the insert region using primers on the vector.

7. Confirm the cloned sequence by Sanger sequencing.

8. PCR-amplify the insert using vector primers containing the $\mathrm{T} 7$ polymerase promoter sequence $^{24,25}$.

NOTE: In this study, the following primers were used: T7-F (5' - TAA TAC GAC TCA CTA TAG GGA GAC TAG TCA TAT GGA T - 3') and T7-R (5'- TAA TAC GAC TCA CTA TAG GGA GAC CCG GGG ATC CGA T - 3') 25 .
9. Purify the PCR product using the PCR purification kit according to the manufacturer's protocol, elute the DNA with $50 \mu \mathrm{L}$ of distilled water, and concentrate the eluted DNA solution to approximately $10 \mu \mathrm{L}$ using a centrifugal evaporator.

10. Synthesize dsRNA by in vitro transcription according to the manufacturer's protocol. Use a total of 1000 ng template DNA and elute synthesized dsRNA with $100 \mu \mathrm{L}$ of elution buffer.

11. Measure the concentration of dsRNA using a spectrophotometer and confirm the quality of dsRNA by electrophoresis on a $1.5 \%$ agarose gel.

NOTE: A single band can be seen when dsRNA synthesis is successful.

12. Dilute dsRNA to $1000 \mathrm{ng} / \mu \mathrm{L}$ with elution buffer and store at $-20^{\circ} \mathrm{C}$ until use.

3. Preparation of injection needles

1. Pull a glass capillary by using a glass needle puller.

2. Place the tip of the pulled capillary onto a doublesided adhesive tape and break the tip of the capillary with forceps.

3. Set the capillary to an injector.

NOTE: It is easier to handle the capillary if you wear nitrile gloves.

4. Load siRNA/dsRNA solution to the prepared capillary.

NOTE: Do not use the capillary repeatedly since the tip of the injected capillary may become clogged with dirt because the larvae collected in the field lived in the mud. 


\section{3. siRNA/dsRNA injection}

NOTE: The procedure is slightly different for damselflies (step 3.1) and dragonflies (step 3.2).

1. Injection to a Zygopteran (damselfly) larva (e.g., I. senegalensis).

1. Anesthetize a larva covered with a wet paper on crushed ice for $50-70$ seconds (Figure 3A-C).

NOTE: Duration of ice-cold anesthesia depends on the condition of the larva; if the larva begins to move after 70 seconds of ice-cold anesthesia, an additional 70 seconds of ice-cold anesthesia is applied. For larvae that rarely move (e.g., $P$. zonata), this procedure is not essential.

2. Attach two pins on both sides of the prothorax and fix the larva to a fixed stand (e.g., a piece of Styrofoam)

(Figure 3D).

NOTE: Adjust the position and number of pins, depending on the place of injection.

3. Stretch the inter-segmental membrane between the 7th and 8th abdominal segment for RNAi in the abdomen or between the prothorax and synthorax (fused mesothorax and metathorax) for RNAi in the thorax using hands and forceps.

4. Keep the inter-segmental membrane stretched by hand.

5. Insert the tip of the prepared capillary into the stretched inter-segmental membrane (Figure 3D, $3 F)$.

6. Inject $1 \mu \mathrm{L}$ of siRNA/dsRNA solution.

2. Injection to an Anisopteran (dragonfly) larva (e.g., $P$. zonata).
1. Wipe the water off the larval surface with a paper towel.

2. If needed, attach two pins on both sides of the prothorax and fix the larva to a fixed stand (e.g., a piece of Styrofoam) (Figure 3H).

NOTE: Adjust the position and number of pins, depending on the place of injection. In the case of $P$. zonata, it is not essential to fix the larvae with pins at this step.

3. Stretch the inter-segmental membrane between the 4th and 5th abdominal segment by hand.

4. Make a small hole with a fine needle in the intersegmental membrane between the 4 th and 5 th abdominal segment.

NOTE: This procedure is necessary for many Anisopteran (dragonfly) species because the intersegmental membrane is too hard to insert a glass capillary directly.

5. Insert the tip of the prepared capillary into the prepared hole (Figure 3I).

NOTE: As shown in Figure $\mathbf{3} \mathbf{H}$, part of the dorsal side of the larvae corresponds to the ventral side of the adult, so the phenotype appears ventrally when treated as shown in Figure 3H-J.

6. Inject $1 \mu \mathrm{L}$ of siRNA/dsRNA solution.

\section{4. in vivo electroporation}

1. If needed, add more pins to fix the larva to a fixed stand (e.g., a piece of Styrofoam).

2. Following the injection of the siRNA/dsRNA solution, apply two droplets of ultrasound gel on the larval surface using forceps. 
3. Place electrodes on the ultrasound gel, with the positive electrode on the side injected with the siRNA/dsRNA solution and a negative electrode on the opposite side (Figure 3E, 3G, 3J).

NOTE: Do not directly touch the epidermis of the larva to avoid the burning effect of electroporation.

4. Generate 10-times electroporation pulses $(280 \mathrm{~ms} / \mathrm{s}$ each) using an electroporator.

NOTE: Adjust the voltage of electroporation, depending on the species, stages and tissues. In this study, $25 \mathrm{~V}$ was applied to $I$. senegalensis and $45 \mathrm{~V}$ to $P$. zonata.

5. Wipe off the remaining gel on the surface with a paper towel.

6. Keep the treated larvae resting on a wet paper towel for approximately one day for recovery and transfer them to a rearing case on the following day.

\section{Site-specific phenotypic analysis}

1. Keep $I$. senegalensis individually in a Petri dish $(5 \mathrm{~cm}$ in diameter) containing approximately $10 \mathrm{~mL}$ of water and a piece of paper towel, and keep $P$. zonata in a plastic cage with a disposable non-woven mesh (eclosion cage ${ }^{14}$ ).

2. For I. senegalensis, after the larvae stop eating, move them individually into a plastic cage with a disposable non-woven mesh.

NOTE: Do not put two or more larvae in the same cage. Otherwise, they will cannibalize each other immediately after they become adults.

3. After the emergence of the adult, observe and photograph the phenotype around the region where the positive electrode was placed for electroporation.
NOTE: The phenotype appears only in patches. Phenotypes are often difficult to recognize immediately after emergence due to incomplete pigmentation.

4. In order to examine the efficiency of RNAi (e.g., quantitative RT-PCR), if the phenotype is visible, dissect the region and compare it with the region without the phenotype.

NOTE: The levels of RNAi phenotype, namely size and location of the cuticle decolorization, often exhibit considerable variation between individuals (see Figure 4).

5. Keep the emerged adults in $100 \% \mathrm{EtOH}$ for future analyses.

NOTE: Body color of Odonata species quickly fades after death, so it is important to store them in ethanol before they die. Ethanol sometimes discolors the insects, in such cases that the insects are frozen before they die.

\section{Representative Results}

We applied the above protocol to electroporation-mediated RNAi targeting MCO2 gene and negative control genes (EGFP for siRNA and bla for dsRNA) (i) in the abdomen of $I$. senegalensis (Figure 4), (ii) in the thorax of I. senegalensis (Figure 5), and (iii) in the abdomen of $P$. zonata (Figure 6). The results of the RNAi experiments are summarized in Table 1. Because negatively charged siRNA/dsRNA is incorporated only into positively charged cells, RNAi phenotypes were observed around the region where the positive electrode was placed for electroporation. 
In both $I$. senegalensis and $P$. zonata, inhibition of melanin pigmentation (i.e., black, brown, and reddish brown) appeared in patches around the region where the positive electrode was placed (white arrowheads and dotted lines in Figure 4, Figure 5, and Figure 6) when MCO2 RNAi was performed in combination with electroporation (Table 1), as previously reported in $N$. pygmaea ${ }^{9}$. By contrast, no phenotypic effects were observed around the electroporation site when the control gene was injected (EGFP siRNA or bla dsRNA) (Figure 4, Figure 5, and Figure 6, Table 1). In addition, injecting the MCO2 gene without electroporation had no effect on adult pigmentation (Figure 4, Table 1), indicating that electroporation is essential for RNAi in Odonata. It should be noted that the blue, green, and yellow colorations are not affected by the RNAi of MCO2 gene that is involved in melanin synthesis in the cuticle, which plausibly reflect the fact that these body colors are attributed to pigment granules present in the epidermal cells that are visible through the transparent cuticle ${ }^{26}$. As shown in Figure 4, no remarkable phenotypic differences were recognized between the individuals subjected to siRNA treatment and dsRNA treatment, whereas considerable variation in size and location of the RNAi phenotype was observed among different individuals subjected to the same RNAi treatment (ex. compare two examples of IsMCO2 dsRNA in Figure 4).

To determine the developmental stage most suitable for the RNAi treatment, we compared the phenotypic consequences of the RNAi treatment at five morphological stages (stage AE) in the final larval instar of $I$. senegalensis (Figure 7A). Inhibition of melanin pigmentation caused by MCO2 RNAi was observed in all emerged adults when injected at the stages $A$ and $B$ (Figure $7 \mathrm{C}$ ). When injected at the stages C and D, suppression of melanin pigmentation was certainly observed in some emerged adults, but other adults exhibited abnormal coloration caused by wounds (Figure 7B). 
A

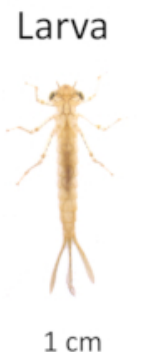

$1 \mathrm{~cm}$

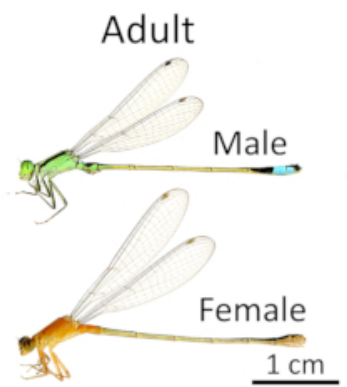

Ischnura senegalensis

(Coenagrionidae, Zygoptera)
B

Larva
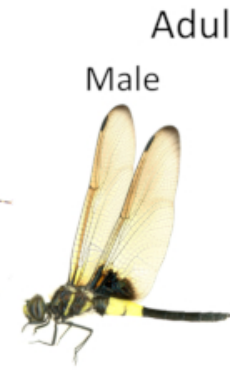

Female

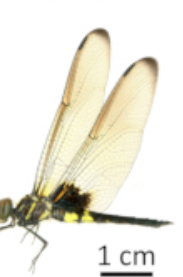

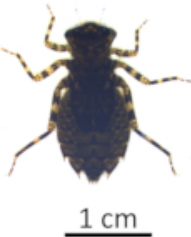

C

1. Preparation of larvae

Pseudothemis zonata

(Libellulidae, Anisoptera)

1.2. Species identification

2. Preparation of dsRNA/siRNA solution and injection needles

3.1. Ice-cold anesthesia

\& dsRNA/siRNA injection $\checkmark$

4. in vivo electroporation $(25 \mathrm{~V})$

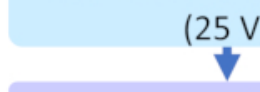

5. Site-specific phenotypic analysis

3.2. Making a small hole

\& dsRNA/siRNA injection

4. in vivo electroporation

$(45 \mathrm{~V})$

Figure 1: Electroporation-mediated RNAi methods in Odonata. A. Ischnura senegalensis (Coenagirionidae) as a representative damselfly species. B. Pseudothemis zonata (Libellulidae) as a representative dragonfly species. C. The overall scheme of the protocols. Blue and orange boxes indicate the protocols for $I$. senegalensis and $P$. zonata, respectively. The purple boxes indicate the common protocols applied to both species. Please click here to view a larger version of this figure. 

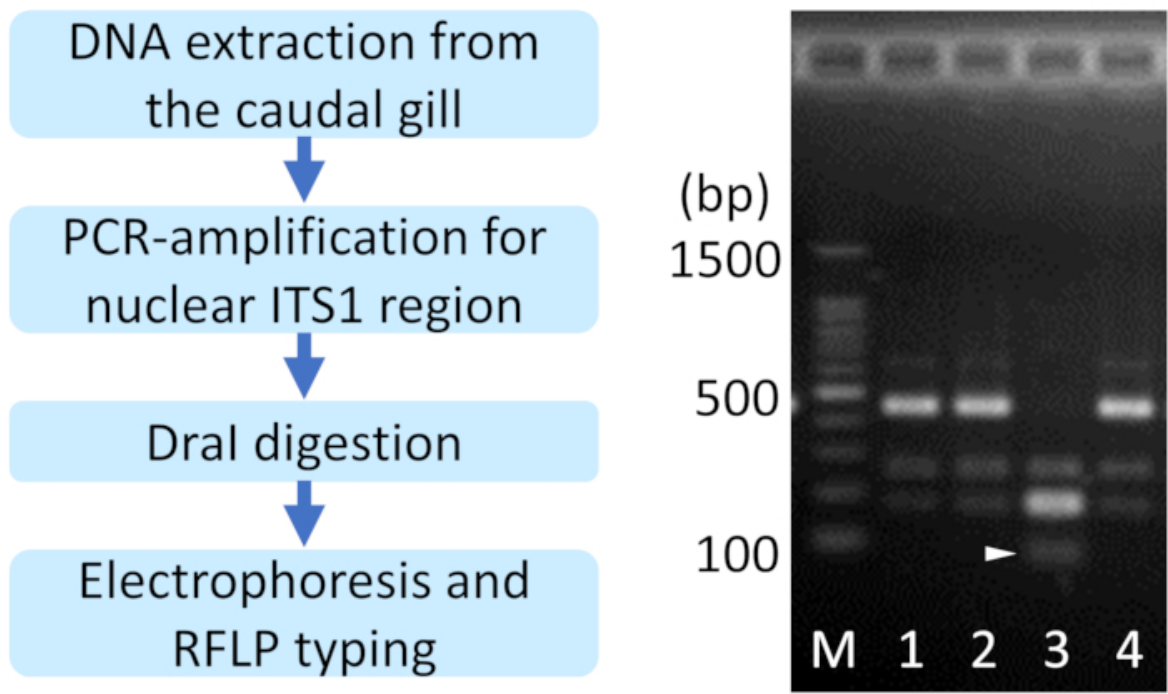

Figure 2: A representative result of restriction fragment length polymorphism (RFLP)-based species identification for Ischnura species. Arrowhead indicates the I. senegalensis-specific band. 1, 2, 4: I. asiatica, 3: I. senegalensis, M: $100-$ base pair ladder marker. Please click here to view a larger version of this figure. 


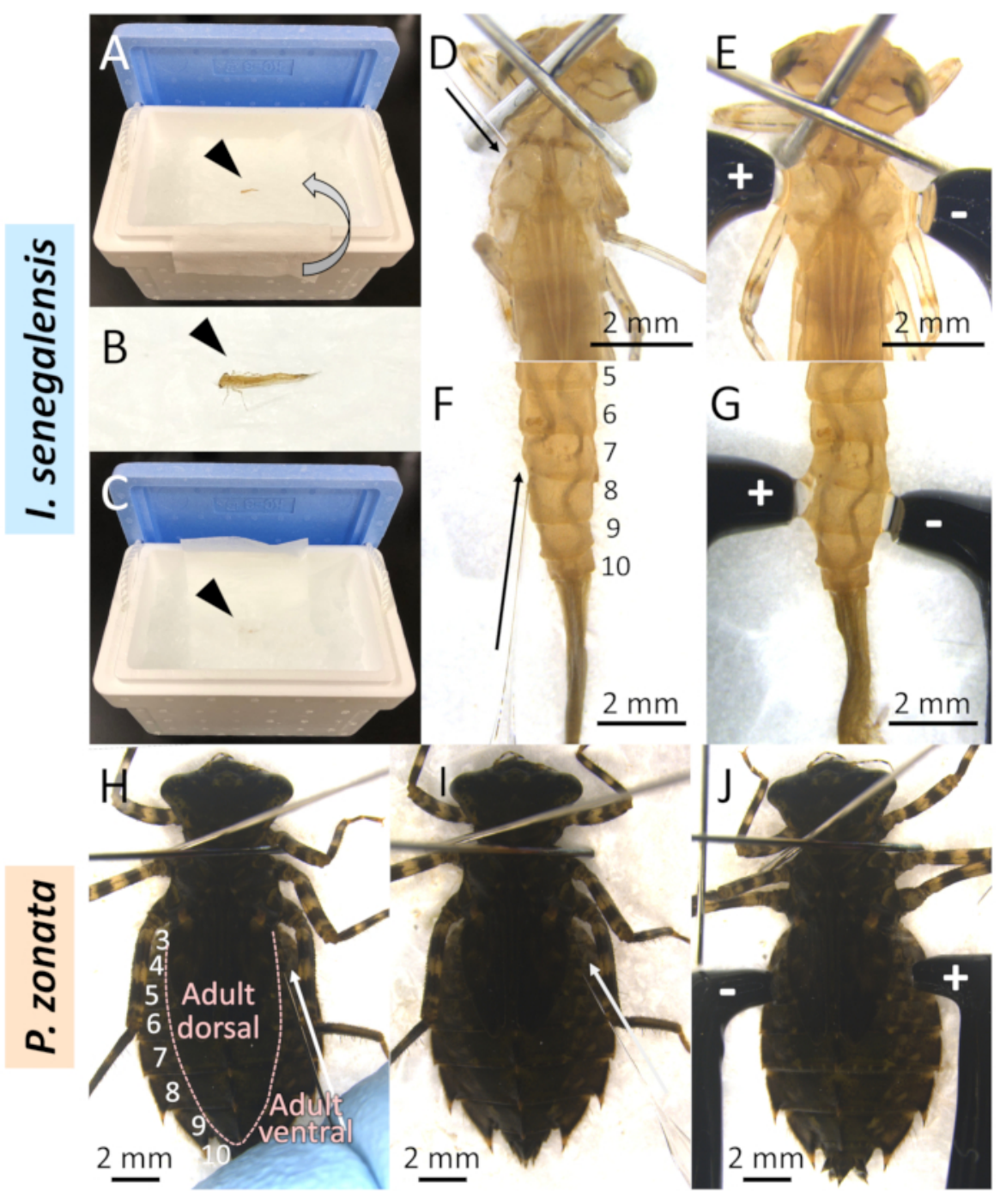

Figure 3: Electroporation-mediated RNAi method in Odonata. A-C. Ice-cold anesthesia of I. senegalensis. Arrowheads indicate a larva. A. Putting a larva on crushed ice with a wet paper. B. Magnified view of a larva on ice. C. A larva covered with a wet paper on ice. D-G. RNAi method for I. senegalensis. D. Injection into the thorax. E. Electroporation on the thorax. F. Injection into the abdomen. G. Electroporation on the abdomen. H-J. RNAi method for P. zonata. H. Making a small hole on the abdomen. I. Injection into the abdomen. J. Electroporation on the abdomen. Arrows indicate the point of making a hole or injection. +, -: Positive/negative side electrodes. Numbers indicate the abdominal segment. Please click here to view a larger version of this figure. 


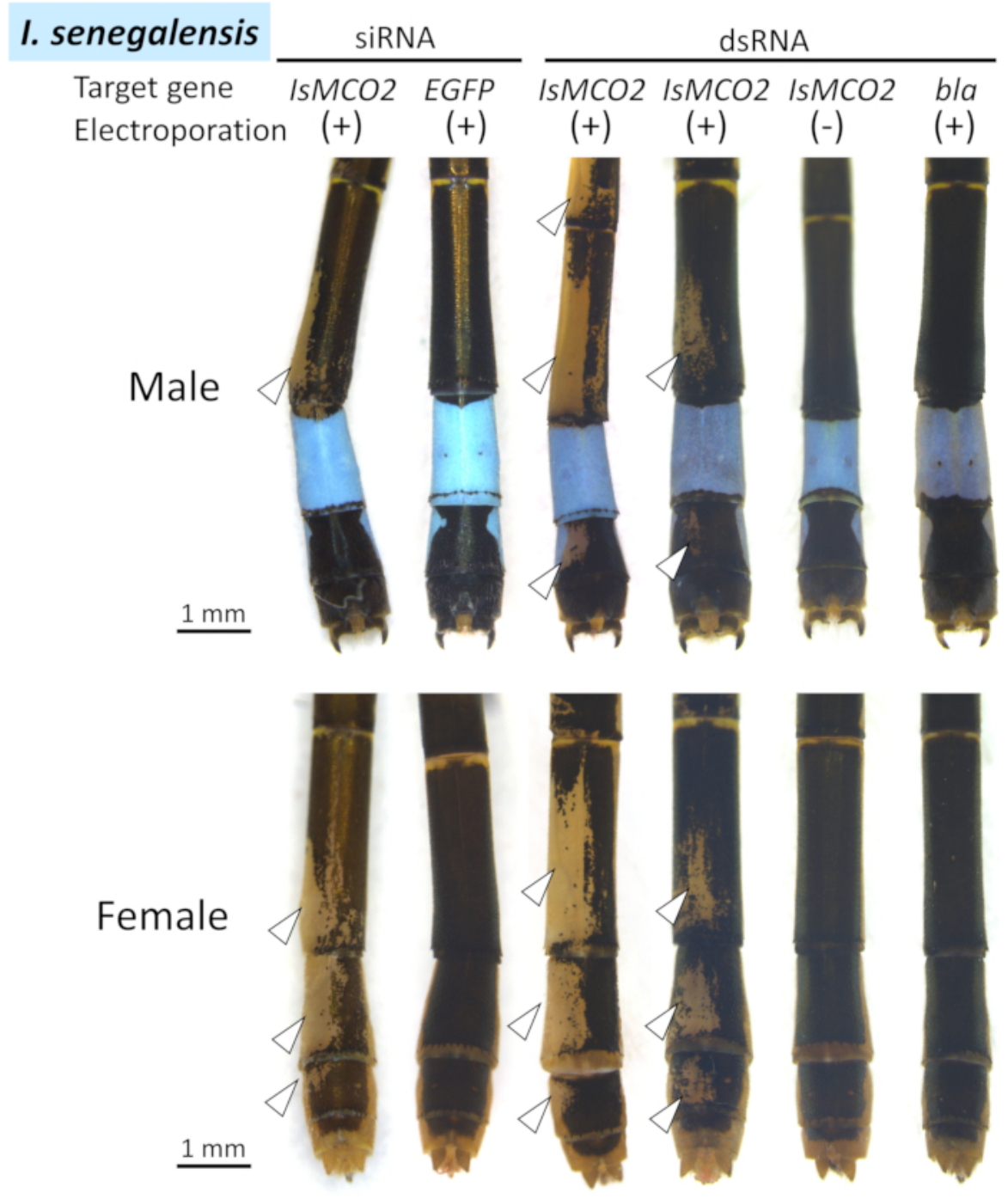

Figure 4: Dorsal views of RNAi phenotypes on the abdomen of $I$. senegalensis. White arrowheads indicate the regions of suppressed melanization. Please click here to view a larger version of this figure. 


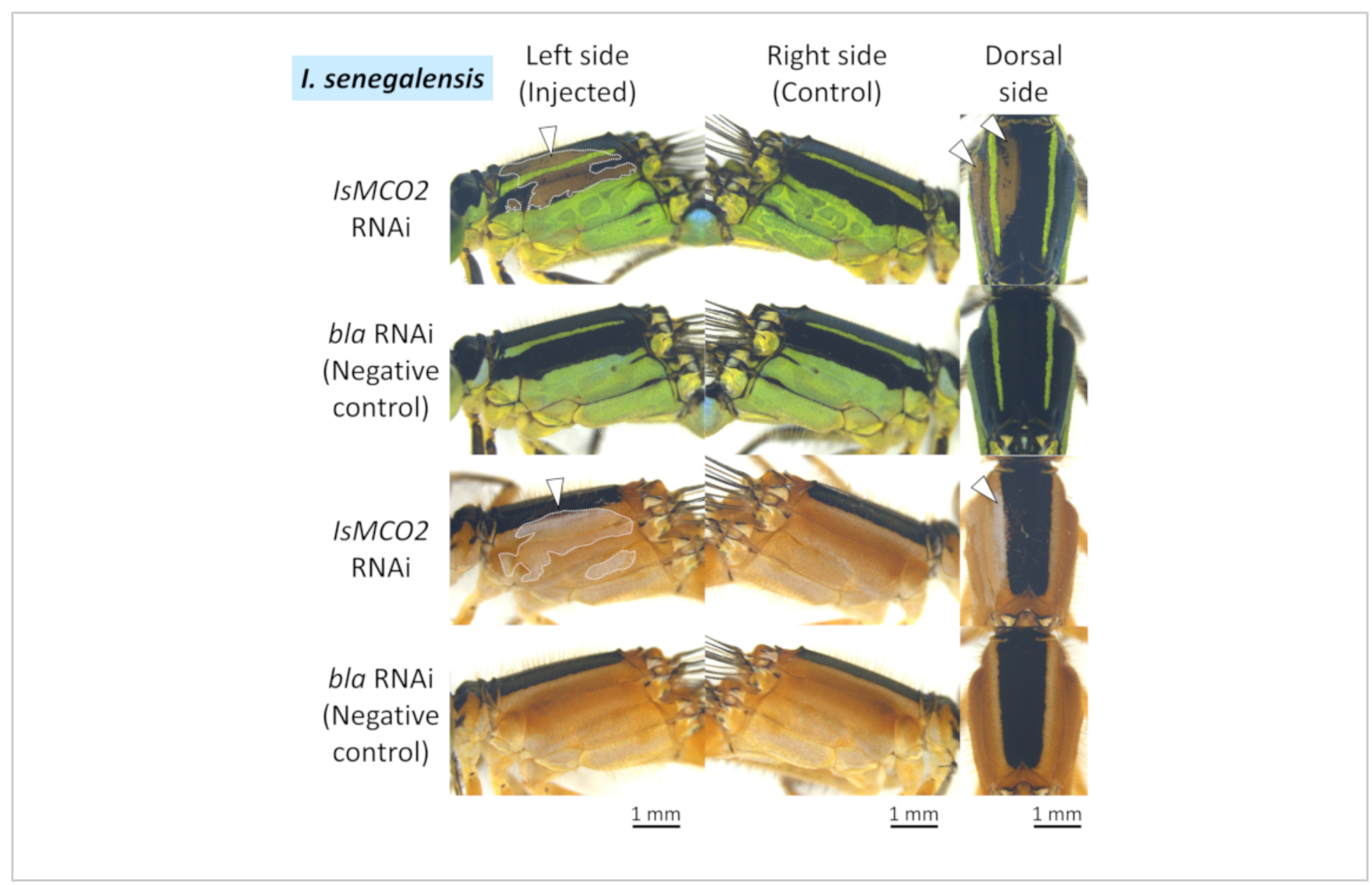

Figure 5: Lateral and dorsal views of RNAi phenotypes on the thorax of $I$. senegalensis. White arrowheads and dotted lines indicate the regions of suppressed pigmentation. Please click here to view a larger version of this figure. 


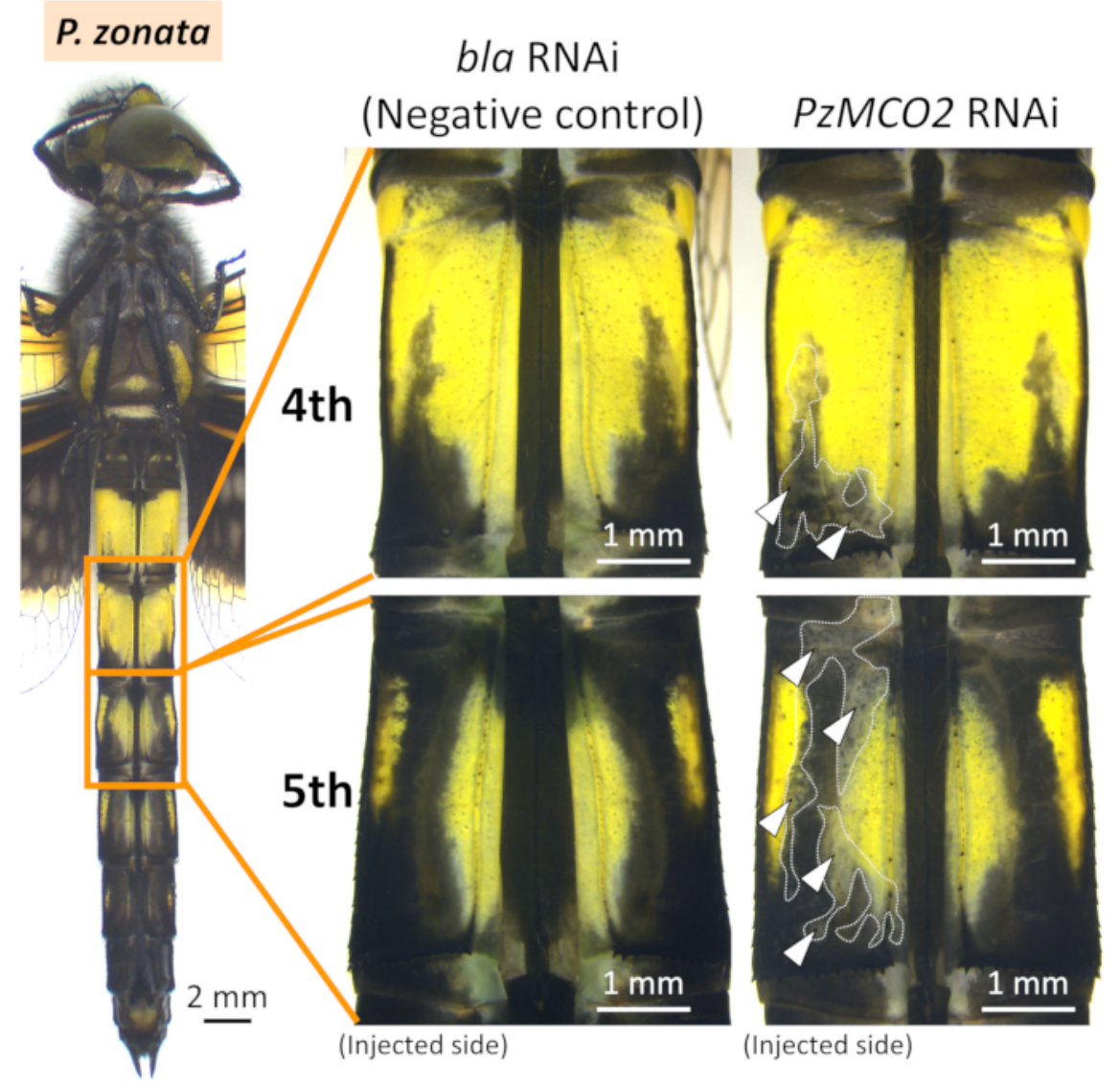

Figure 6: Ventral views of RNAi phenotypes in the abdomen of $\boldsymbol{P}$. zonata. White arrowheads and dotted lines indicate the regions of suppressed melanization. Please click here to view a larger version of this figure. 


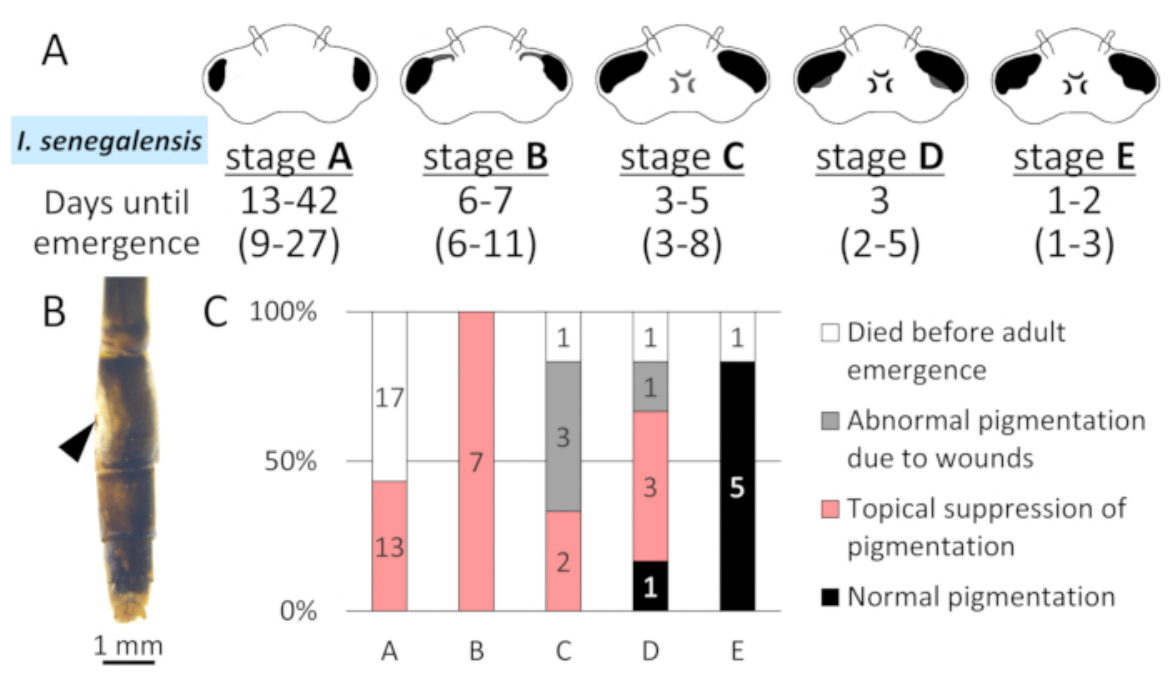

Figure 7: Stage dependent IsMCO2 RNAi effects during the final larval instar of I.senegalensis. A. Morphological changes in the compound eyes at five morphological stages (stage A-E) and the number of days to adult emergence in this study. Numbers in parentheses are from previous report ${ }^{14}$. B. Abnormal pigmentation due to wounds. Arrowhead indicates electroporation site. C. The effect of RNAi at five morphological stages on adult pigmentation in $I$. senegalensis. The number on the bar indicates the number of individuals. Please click here to view a larger version of this figure. 


\begin{tabular}{|c|c|c|c|c|c|c|c|c|c|c|}
\hline Species & \multicolumn{7}{|c|}{ I.senegalensis } & \multicolumn{3}{|c|}{ P.zonata } \\
\hline Injected region & \multicolumn{5}{|c|}{ Abdomen } & \multirow{2}{*}{\multicolumn{2}{|c|}{$\begin{array}{l}\text { Thorax } \\
\text { dsRNA }\end{array}$}} & \multirow{2}{*}{\multicolumn{3}{|c|}{$\frac{\text { Abdomen }}{\text { dsRNA }}$}} \\
\hline siRNA/dsRNA & \multicolumn{2}{|c|}{ siRNA } & \multicolumn{3}{|c|}{ dsRNA } & & & & & \\
\hline Target gene & IsMCO2 & $E G F P$ & IsMCO2 & IsMCO2 & bla & IsMCO2 & bla & PzMCO2 & PzMCO2 & bla \\
\hline Electroporation & + & + & + & - & + & + & + & + & - & + \\
\hline Injected larvae & 22 & 25 & 30 & 6 & 53 & 12 & 20 & 17 & 5 & 9 \\
\hline Emerged adults & 7 & 6 & 13 & 4 & 40 & 11 & 14 & 11 & 2 & 5 \\
\hline $\begin{array}{c}\text { Adults with } \\
\text { less pigmented } \\
\text { regions (ratio) }\end{array}$ & $\begin{array}{c}7 \\
(100 \%)\end{array}$ & $\begin{array}{c}0 \\
(0 \%)\end{array}$ & $\begin{array}{c}13 \\
(100 \%)\end{array}$ & $\begin{array}{c}0 \\
(0 \%)\end{array}$ & $\begin{array}{c}0 \\
(0 \%)\end{array}$ & $\begin{array}{c}10 \\
(91 \%)\end{array}$ & $\begin{array}{c}0 \\
(0 \%)\end{array}$ & $\begin{array}{c}11 \\
(100 \%)\end{array}$ & $\begin{array}{c}0 \\
(0 \%)\end{array}$ & $\begin{array}{c}0 \\
(0 \%)\end{array}$ \\
\hline
\end{tabular}

Table 1. The effect of RNAi on adult pigmentation in $I$. senegalensis and $P$. zonata. Results at stage A are shown in $I$. senegalensis. IsMCO2: multicopper oxidase 2 gene of I. senegalensis, EGFP: Enhanced green fluorescent protein gene, bla: beta lactamase gene from pGEM-T Easy Vector, PzMCO2: multicopper oxidase 2 gene of $P$. zonata. The results for the control genes represent the total number of experiments the authors have conducted to date.

\section{Discussion}

\section{Lethality of RNAi treatment}

We found that the lethality of the RNAi treatment depends strongly on the rearing history and condition of the Odonata larvae. The larvae soon after collection in the field are generally healthy and exhibit low mortality rates after the electroporation-mediated RNAi treatment. By contrast, the larvae reared in the laboratory for a long period of time (e.g., one month) tend to suffer low success rates of adult emergence. In I. senegalensis, instead of the larvae collected in the field, the larvae reared in the laboratory from eggs can be used ${ }^{14}$, but the success rates of RNAi using the laboratory-reared larvae tend to be considerably lower (many individuals died during metamorphosis) than those using the field-collected larvae. In addition, frequent larval feeding and clean water rearing are important for increasing the success rates of adult emergence and reducing the lethality of the RNAi treatment.

\section{Efficiency of RNAi treatment}

As described above, the levels of RNAi phenotype, namely size and location of the cuticle decolorization, often exhibited considerable variation between individuals subjected to the same RNAi treatment (e.g., Figure 4), but the levels of the phenotypic penetrance seem to be remarkably different between the Odonata species. The observed phenotypic regions were larger and more prominent in I. senegalensis (Figures 4-5) than in P. zonata (Figure 6) and N. pygmaea ${ }^{9}$. This difference may be due to the thickness of the cuticle on the larval surface, considering that the cuticle of $I$. senegalensis is thinner than the cuticle of $P$. zonata and $N$. pygmaea). As far as we examined, no clear difference was 
recognized between the effects of siRNA and dsRNA (Figure 4, Table 1).

\section{Appropriate developmental stage for RNAi}

It should be noted that proper larval staging is important for performing RNAi efficiently. Inhibition of adult pigmentation was caused by MCO2 RNAi before the stage D (approximately 3 days before adult emergence), which is consistent with the previous report on $N$. pygmaea ${ }^{9}$. The RNAi phenotypes observed when injected at the stages C and $\mathrm{D}$ were less conspicuous than those treated at the stages $A$ and $B$, which indicate that the stages $C$ and $D$ may be too late to sufficiently suppress the gene expression. The appropriate timing for RNAi treatment depends on the timing of gene expression, and MCO2 gene exhibits transiently high expression during adult emergence ${ }^{9}$, as in other insects ${ }^{17,18}$. In the stinkbug Plautia stali, RNAi knockdown of MCO2 gene was observed from day 4 onwards after injection ${ }^{27}$, which is consistent with the present results.

Our previous study on $I$. senegalensis showed that, after the stage $B$, days to adult emergence exhibit relatively small variation among the majority of final instar larvae, suggesting that the stage B may correspond to the onset of the process toward adult emergence, after which the developmental processes for metamorphosis proceed in a prefixed and coordinated manner ${ }^{14}$. Morphological abnormalities caused by wounds were often observed when the larvae were RNAitreated at the stages $C$ and $D($ Figure $7 \mathbf{B}, 7 \mathbf{C})$. This is likely to be associated with a dramatic progression of metamorphosis during these stages, suggesting that RNAi treatment should be avoided from the stage $\mathrm{C}$ and on. In summary, we recommend that final instar larvae at the stage A or B (or at the stage before the larval wings expand significantly) should be used for RNAi experiments.

\section{Usefulness and superiority of electroporation-mediated RNAi method}

The conventional RNAi is a simple and powerful experimental method, but some insect lineages like butterflies ${ }^{10}$, aphids ${ }^{28}$ and dragonflies ${ }^{9}$ exhibit low RNAi efficiency, for which establishment of gene function analysis is a major challenge. In this study, we found that electroporation-mediated RNAi can induce local gene suppression in dragonflies with almost $100 \%$ efficiency, at least in epidermis, if treated at appropriate developmental stages (Table 1). Recently, CRISPR/Cas9based gene knockouts have been successfully applied to a variety of insects, providing a powerful molecular genetic tool for non-model organisms ${ }^{29}$. Here, however, we point out that CRISPR/Cas9 is certainly great but the electroporationmediated RNAi method may be superior to CRISPR/Cas9 in some respects

Firstly, in the electroporation-mediated RNAi method, the body region where RNAi phenotypes appear can be easily controlled experimentally by the position of the positive electrode upon electroporation. In addition, since the region where the gene expression is suppressed is limited around the region where the positive electrode was placed, the RNAi phenotypes can be easily compared with the control phenotypes side by side in the same individual. Secondly, compared to CRISPR/Cas9 method in which injected eggs have to be reared to adulthood to observe the knockout phenotypes, the electroporation-mediated RNAi is superior in that the gene knockdown phenotypes can usually be observed in much shorter time. For example, it takes three to four months for $I$. senegalensis and one to two years for $P$. zonata from eggs to adults ${ }^{14,30}$. However, in order to observe 
RNAi phenotypes within the adult epidermis, it takes less than one month from dsRNA injection into final instar larvae at the stage $B$ to adult emergence for both $I$. senegalensis and $P$. zonata (Figure 7). Thirdly, the electroporation-mediated RNAi method entails dsRNA injection into large larvae, which is easier than microinjection into tiny eggs required for CRISPR/ Cas9 method. In addition, the electroporation-mediated RNAi is applicable to insect species whose newly laid eggs are difficult to collect. For example, females of $P$. zonata lay eggs onto floating plants on water surface during flight, and thus it is difficult to collect their eggs both in the field and in the lab. Hence, we expect that this protocol may be generally applicable to non-model organisms in which the conventional RNAi method does not work efficiently.

\section{Disclosures}

The authors have nothing to disclose.

\section{Acknowledgments}

We thank Minoru Moriyama for technical advice and supports, Bin Hirota and Ryutaro Suzuki for collecting Odonata larvae, and Misa Shinya for helpful comments on the manuscript. This work was supported by JSPS KAKENHI Grant Numbers JP18J21561 to GO and JP18H02491, JP18H04893, JP19H03287, and JP2OH04936 to RF.

\section{References}

1. Misof, B. et al. Phylogenomics resolves the timing and pattern of insect evolution. Science. 346 (6210), 763-767 (2014).

2. Wipfler, B. et al. Evolutionary history of Polyneoptera and its implications for our understanding of early winged insects. Proceedings of the National Academy of Sciences of the United States of America. 116 (8), 3024-3029 (2019).

3. Corbet, P. S. Dragonflies, Behavior and Ecology of Odonata. Cornell University Press, Ithaca, NY (1999).

4. Futahashi, R. Color vision and color formation in dragonflies. Current Opinion in Insect Science. 17, 32-39 (2016).

5. Futahashi, R. Diversity of UV reflection patterns in Odonata. Frontiers in Ecology and Evolution. 8, 201 (2020).

6. Córdoba-Aguilar, A. Dragonflies and damselflies. Model organisms for ecological and evolutionary research. Oxford University Press, Oxford (2008).

7. Bybee, S. et al. Odonata (dragonflies and damselflies) as a bridge between ecology and evolutionary genomics. Frontiers in Zoology. 13, 46 (2016).

8. Linz, D. M., Clark-Hachtel, C. M., Borràs-Castelles, Tomoyasu, Y. Larval RNA interference in the red flour beetle, Tribolium castaneum. Journal of Visualized Experiments. 92, 52059 (2014).

9. Okude, G. et al. Electroporation-mediated RNA interference reveals a role of multicopper oxidase 2 gene in dragonfly's cuticular pigmentation. Applied Entomology and Zoology. 53 (3), 379-387 (2017).

10. Terenius, O. et al. RNA interference in Lepidoptera: An overview of successful and unsuccessful studies and implications for experimental design. Journal of Insect Physiology. 57, 231-245 (2011).

11. Ando, T., Fujiwara, H. Electroporation-mediated somatic transgenesis for rapid functional analysis in insects. Development. 140, 454-458 (2013). 
12. Nishikawa, H. et al. A genetic mechanism for femalelimited Batesian mimicry in Papilio butterfly. Nature Genetics. 47 (4), 405-409 (2015).

13. Osanai-Futahashi, M. et al. Positional cloning of a Bombyx pink-eyed white egg locus reveals the major role of cardinal in ommochrome synthesis. Heredity. 116 (2), 135-145 (2016).

14. Okude, G., Futahashi, R., Tanahashi, M., Fukatsu, T. Laboratory rearing system for Ischnura senegalensis (Insecta: Odonata) enables detailed description of dragonfly's larval development and morphogenesis. Zoological Science. 34 (5), 386-397 (2017).

15. Ozono, A., Kawashima, I., Futahashi, R. Dragonflies of Japan (3rd edition). Bunichi-Sogo Syuppan, Co Ltd, Tokyo (2017).

16. Ozono, A., Kawashima, I., Futahashi, R. The Handbook of Japanese Aquatic Insects. Volume 3: Dragonfly larvae. Bunichi-Sogo Syuppan, Co Ltd, Tokyo (2019).

17. Arakane, Y., Noh, M. Y. , Asano, T., Kramer, K. J. Tyrosine metabolism for insect cuticle pigmentation and sclerotization. Extracellular Composite Matrices in Arthropods. (eds Cohen, E., \& Moussian, B.) 165-220, Springer (2016).

18. Asano, T. et al. Mini-review an insect-specific system for terrestrialization: Laccase-mediated cuticle formation. Insect Biochemistry and Molecular Biology. 108, 61-70 (2019).

19. Futahashi, R., Okude, G., Sugimura, M., Ugai, S. Interspecific hybrids in Japanese Odonata. Tombo. 60, $1-49$ (2018).

20. Okude, G., Fukatsu, T., Futahashi, R. Interspecific crossing between blue-tailed damselflies Ischnura elegans and $I$. senegalensis in the laboratory. Entomological Science. 23 (2), 165-172 (2020).

21. Naito, Y., Yoshimura, J., Morishita, S., Ui-Tei, K. siDirect 2.0: updated software for designing functional siRNA with reduced seed-dependent off-target effect. BMC Bioinformatics. 10, 392 (2009).

22. Yamaguchi, J., Mizoguchi, T., Fujiwara, H. siRNAs induce efficient RNAi response in Bombyx mori embryos. PLoS ONE. 6 (9), e25469 (2011).

23. Untergasser, A. et al. Primer3--new capabilities and interfaces. Nucleic Acids Research. 40 (15), e115 (2012).

24. Futahashi, R. Whole-mount in situ hybridization of sectioned tissues of species hybrids to detect cisregulatory changes in gene expression pattern. Methods in Molecular Biology. 772, 319-328 (2011).

25. Matsuura, Y., Kikuchi, Y., Miura, T., Fukatsu, T. Ultrabithorax is essential for bacteriocyte development. Proceedings of the National Academy of Sciences of the United States of America. 112 (30), 9376-9381 (2015).

26. Henze, M. J., Lind, O., Wilts, B. D., Kelber, A. Pterinpigmented nanospheres create the colours of the polymorphiic damselfly Ischnura elegans. Journal of The Royal Society Interface. 16, 20180785 (2019).

27. Nishide, Y. et al. Diversity and function of multicopper oxidase genes in the stinkbug Plautia stali. Scientific Reports. 10 (1), 3464 (2020).

28. Christiaens, O., Swevers, L., Smagghe, G. DsRNA degradation in the pea aphid (Acyrthosiphon pisum) associated with lack of response in RNAi feeding and injection assay. Peptides. 53, 307-314 (2014). 
29. Sun, D., Guo, Z., Liu, Y., Zhang, Y. Progress and prospects of CRISPR/Cas systems in insects and other arthropods. Frontiers in Physiology. 8, 608 (2017).

30. Miyakawa, K. A study of the life-history of Pseudothemis zonata (Burm.) (Odonata, Libellulidae) II. Immature stage. Kontyû. 37 (4), 409-422 (1969). 